\title{
Formación y aplicación didáctica de las TIC en proyectos para trabajar la lengua y la literatura
}

\author{
Pilar Garcia Vidala, Alícia Martí Climent ${ }^{\text {b }}$ \\ aUniversitat de València, pilar.garcia-vidal@uv.es \\ bUniversitat de València, alicia.marti@uv.es
}

\begin{abstract}
In order to study the ICT training received by university students and the didactic application that the future teachers make of these kind of resources, we have made a qualitative analysis based on questionnaires made to the students of the Master's Degree in Secondary School Training at the University of Valencia from 2011 to 2016. We consider that students are continually increasing their knowledge of ICT technology. However, we think that they still need to use ICT with a didactic purpose in the academic work. This research shows both the ICT knowledge of these students and it's implementation considered to be useful to work in language and literature projects in Secondary Education.
\end{abstract}

Keywords: Project work, ICT, teacher training, secondary education, language and literatura teaching.

\footnotetext{
Resumen

Con el fin de indagar en la formación en TIC que han recibido los estudiantes universitarios y en la aplicación didáctica que hacen de este tipo de recursos los futuros docentes, hemos realizado un análisis cualitativo a partir de cuestionarios realizados al alumnado del Máster de profesor/a de Secundaria de la Universitat de València desde el curso 2011-12 hasta el 2015-16. Partimos de la hipótesis que los estudiantes conocen cada vez más recursos TIC pero les falta práctica en su uso en el trabajo académico con una finalidad didáctica. El presente análisis muestra el grado de conocimiento de las TIC de estos estudiantes, así como la implementación de determinadas TIC que han considerado útiles para trabajar en proyectos de lengua y literatura en Secundaria.
}

Palabras clave: Trabajo por proyectos, TIC, formación del profesorado, enseñanza secundaria, didáctica de la lengua y la literatura. 


\section{Introducción}

La innovación y la mejora de las prácticas educativas mediante las tecnologías depende del buen uso en su aplicación didáctica. En este trabajo presentamos una selección de recursos TIC utilizados habitualmente en el trabajo académico por los estudiantes universitarios con el objetivo de conocer cuáles son los que utilizan con más frecuencia y el rendimiento que consideran que pueden obtener para conseguir un trabajo más eficaz. El estudio se ha realizado a partir de cuestionarios planteados a los alumnos sobre el uso que hacen de las TIC y sobre las ventajas e inconvenientes que encuentran en el trabajo académico. Además, se ha analizado el uso que han hecho de las TIC en proyectos de trabajo sobre lengua y literatura dirigidos al alumnado de secundaria. De esta forma, hemos comprobado cómo determinados recursos TIC pueden contribuir a mejorar la escritura académica de forma que el aprendizaje lingüístico resulte más eficaz y motivador para el alumnado.

Los estudios sobre alfabetización académica permiten conocer las dificultades del alumnado para interpretar y producir textos académicos. Carlino (2013) indica que alfabetizar académicamente equivale a ayudar a participar en prácticas discursivas contextualizadas. Se trata de favorecer el aprendizaje de las "literacidades" 1 académicas, es decir, la participación de los alumnos en sus culturas escritas.

Es cada vez más evidente, tal como establece el currículum oficial en las diferentes etapas educativas, que el alumnado debería saber utilizar las TIC como instrumentos habituales de trabajo: conocer diferentes herramientas para bajar, colgar, enviar y compartir diferentes tipos de documentos; usar vídeos educativos como recurso formativo e interactivo entre alumnado y profesorado; hacer uso de los enlaces; elaborar y publicar noticias; emplear la prensa digital como recurso y estrategia para aprender a aprender, fomentando la selección y el procesamiento de la información; interactuar, relacionarse con los otros, cooperar, gestionar y resolver conflictos, etc.

Tomaremos como ejemplo el trabajo realizado por los estudiantes del Máster Universitario en Profesor/a de Educación Secundaria basado en proyectos para enseñar y aprender lengua y literatura con el uso de las TIC. En este trabajo se emplea el modelo de enseñanza de composición escrita conocido como secuencia didáctica (SD) que se articula alrededor de la composición de un género discursivo. Los objetivos de aprendizaje propuestos y las

${ }^{1}$ El término Literacy, traducido como "literacidad" por Carlino (2013) se refiere a la formación continua en lectura y escritura que nos convierte en persones letradas que pueden hacer uso de habilidades discursivas de forma activa y ajustada a las exigencias de los contextos en que participan (Castelló, 2014).

\begin{tabular}{|l|l|l|l|l}
\hline (cc) EY-NC-ND 2017, Universitat Politècnica de València &
\end{tabular} 
actividades para conseguirlos suponen diferentes procesos de elaboración lingüística: planificación, textualización y revisión.

En este tipo de proyectos, los alumnos tienen que reflexionar sobre la manera de usar la lengua para conseguir hacer un determinado producto: un programa de radio, un periódico, un videolit, etc. La introducción de las TIC permite considerar las formas lingüísticas desde una perspectiva más funcional puesto que supone plantear el uso de la lengua en determinadas situaciones de comunicación y con unos géneros discursivos concretos que requieren la interacción entre los alumnos, para coordinarse y decidir los recursos que emplearán, y tener en cuenta los receptores del trabajo puesto que se trata de proyectos que presuponen una difusión en la red mediante las TIC. Los géneros discursivos son instrumentos de participación en la actividad social y suponen aprender a usar la lengua de forma adecuada en cada uno de los ámbitos de la actividad humana (Camps, Castelló, 2013). En este caso, la actividad discursiva que se da en el entorno académico se refiere a las situaciones en las que los alumnos de secundaria tienen que aprender a usar el lenguaje en diferentes situaciones comunicativas. Tenemos que tener en cuenta que, junto a géneros marcadamente académicos, hay otros que responden a situaciones comunicativas diferentes que han ido apareciendo con la incorporación de las nuevas tecnologías, como la participación en las redes sociales, la elaboración de un blog o página web.

\section{Objetivos}

Este trabajo tiene como principales objetivos:

- Conocer la formación recibida y el uso de las TIC de los estudiantes universitarios en los trabajos académicos

- Identificar las principales ventajas e inconvenientes que encuentra el alumnado en el uso de las TIC y si hay cambios significativos en los últimos cursos

- Valorar la implementación y la repercusión que han tenido estas herramientas en los proyectos para trabajar la lengua y la literatura en Secundaria.

\section{Desarrollo de la innovación}

En la metodología utilizada para este estudio se ha realizado un análisis cualitativo a partir de la revisión de los cuestionarios iniciales y finales contestados por un centenar de alumnos del Máster de profesor/a de Secundaria de la Universitat de València desde el curso 2011-12 hasta el 2015-16. De esta forma, se ha comprobado qué tipo de recursos TIC conocen y usan los alumnos con más frecuencia y cómo han ido cambiando y modificándose durante los últimos años a lo largo de su formación académica y en su 
aplicación en el trabajo académico. Esta evolución nos permite conocer las herramientas TIC que los estudiantes consideran más efectivas.

En cuanto a la formación recibida y el uso de las TIC, hemos destacado lo que indica el alumnado de forma recurrente respecto a la frecuencia de conexión a Internet, el conocimiento que tiene de las diferentes TIC y las ventajas e inconvenientes que encuentra en su utilización en el trabajo académico.

Además, se han identificado las TIC utilizadas en proyectos para trabajar la lengua y la literatura por estos mismos alumnos durante los cinco cursos indicados. Con estos datos hemos podido comprobar el tipo de recursos TIC que el alumnado considera que son más eficaces para la aplicación didáctica de determinados contenidos de lengua y literatura del currículum de Secundaria ya que les han facilitado la planificación, elaboración y presentación de proyectos en una gran diversidad de géneros académicos.

\section{Resultados}

A partir del análisis de los cuestionarios realizados por los alumnos, se han detectado los siguientes resultados:

\subsection{Conocimiento de las TIC y uso en la formación académica anterior}

Sobre la frecuencia de conexión a la red, los alumnos del máster contestan un cuestionario inicial sobre el conocimiento, el grado de dominio y el uso de las TIC. El 78,66\% del alumnado afirma conectarse a Internet una, dos o tres veces al día. Predomina la opción de 2 o 3 veces al día en todos los cursos y es el alumnado de los últimos cursos el que emplea con más frecuencia Internet.

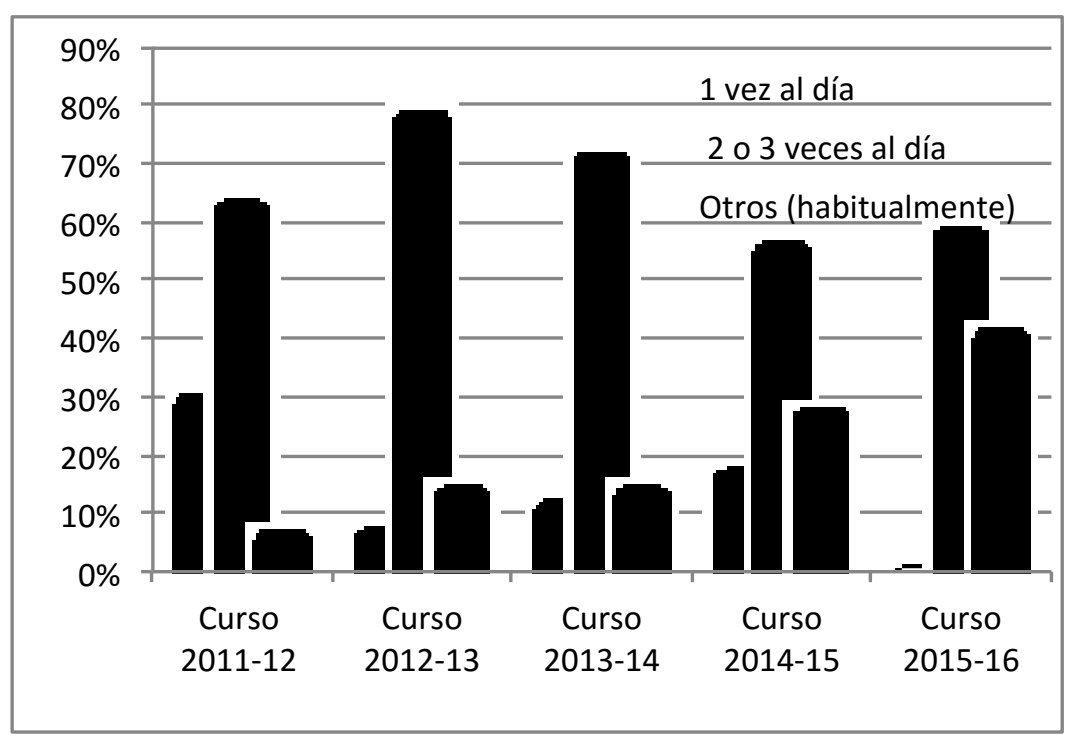

Gráfica 1: Frecuencia de conexión a la red

\begin{tabular}{|l|l|l|l}
\hline (cc) EY-NC-ND 2017, Universitat Politècnica de València &
\end{tabular} 
En relación al conocimiento de las TIC, también se manifiesta una progresión cronológica. Los porcentajes de conocimiento son mucho más altos los últimos cursos, por ejemplo en el curso 2015-16 la mayor parte del alumnado afirma conocer la mayoría de las herramientas planteadas.

Tabla 1. Conocimiento de las TIC

\begin{tabular}{|c|c|c|c|c|c|}
\hline CURSO & 2011-12 & 2012-13 & 2013-14 & 2014-15 & 2015-16 \\
\hline Blog & $96,40 \%$ & $100 \%$ & $97,60 \%$ & $100 \%$ & $100 \%$ \\
\hline Wiki & $64,30 \%$ & $57,10 \%$ & $64,30 \%$ & $72,40 \%$ & $62,10 \%$ \\
\hline Drive & $39,30 \%$ & $7,10 \%$ & $35,70 \%$ & $82,80 \%$ & $89,70 \%$ \\
\hline Box & $7,10 \%$ & $50 \%$ & $71,40 \%$ & $34,50 \%$ & $65,50 \%$ \\
\hline Moodle & $14,30 \%$ & $21,40 \%$ & $33,30 \%$ & $27,60 \%$ & $58,60 \%$ \\
\hline Prezi & & $35,70 \%$ & $38,10 \%$ & $62,10 \%$ & $79,30 \%$ \\
\hline Movie Maker & & & $61,90 \%$ & $72,40 \%$ & $82,80 \%$ \\
\hline Slideshire & $10,70 \%$ & $42,90 \%$ & $28,60 \%$ & $58,60 \%$ & $62,10 \%$ \\
\hline Audacity & & & $26,20 \%$ & $41,40 \%$ & $27,60 \%$ \\
\hline Webquest & $28,60 \%$ & $14,30 \%$ & $16,70 \%$ & $17,20 \%$ & $13,80 \%$ \\
\hline CMapTools & $7,10 \%$ & $0 \%$ & $7,10 \%$ & $0 \%$ & $13,80 \%$ \\
\hline Hot Potatoes & & & $2,40 \%$ & $3,40 \%$ & $3,40 \%$ \\
\hline Glogster & & & $7,10 \%$ & $3,40 \%$ & $0 \%$ \\
\hline
\end{tabular}

No obstante, los alumnos manifiestan que, durante su formación académica, en la mayor parte de las materias no han utilizado nunca ningún recurso TIC. Aún así, observamos que aumenta considerablemente en los últimos cursos el uso y la variedad de las TIC empleadas: si en el curso 2011-12 predomina el blog y los alumnos usan 8 herramientas diferentes, en el 2015-16 predomina también de forma destacada el drive y, además, en este último curso se emplean 12 herramientas TIC diferentes.

\subsection{Ventajas e inconvenientes sobre el uso de las TIC}

En los últimos cursos aumentan las valoraciones positivas y disminuyen las negativas sobre el uso de las TIC. La mayoría de los cursos coinciden en las siguientes ventajas: utilidad, inmediatez/rapidez, fácil acceso, trabajo colaborativo, coordinación, facilidad para compartir, trabajo a distancia, variedad de información, comodidad y facilidad. Como inconvenientes, en la mayoría de los cursos se apunta la escasa formación en TIC, la 
dependencia del acceso a Internet, la carencia de infraestructuras y la pérdida de proximidad personal.

\subsection{Implementación de las TIC en proyectos para trabajar la lengua y la literatura}

En relación a las herramientas TIC que emplean los alumnos desde el curso 2011-12 hasta el 2015-16 en los trabajos por proyectos con diferentes géneros académicos ${ }^{2}$, se constata el aumento de las TIC empleadas en cada curso puesto que los alumnos conocen más cantidad y variedad de ellas, y encuentran que son más efectivas para trabajar en los proyectos.

Tabla 2. Herramientas TIC empleadas en los trabajos por proyectos de los diferentes cursos. Indicamos en negrita las que se van incorporando en cada curso

\begin{tabular}{|c|c|c|c|c|}
\hline $2011-12$ & $2012-13$ & $2013-14$ & $2014-15$ & $2015-16$ \\
\hline $\begin{array}{l}\text { Google } \\
\text { Maps }\end{array}$ & Blog & Google Maps, Blog & Google Maps, Blog & Google Maps, Blog, \\
\hline $\begin{array}{l}\text { Editor de } \\
\text { vídeo }\end{array}$ & $\begin{array}{l}\text { Editor de } \\
\text { vídeo }\end{array}$ & Editor de vídeos & Editor de vídeo & Editor de vídeo, \\
\hline $\begin{array}{l}\text { Editor de } \\
\text { fotografia }\end{array}$ & Webquest & $\begin{array}{l}\text { Webquest, editor de } \\
\text { fotos }\end{array}$ & Webquest, Youtube & Youtube \\
\hline \multirow[t]{14}{*}{ Wiki } & & Wiki & Google Drive, & Google Drive \\
\hline & & Editor d'àudio & Editor d'àudio & Editor d'àudio \\
\hline & & Viquipèdia & Viquipèdia & Viquipèdia, \\
\hline & & Editor d'àudio & Editor d'àudio & Editor d'àudio \\
\hline & & $\begin{array}{l}\text { Línia del temps } \\
\text { digital }\end{array}$ & Línia del temps digital & Instagram \\
\hline & & Facebook & Facebook & Videoconferència \\
\hline & & Podcast & Podcast & Videoquiz \\
\hline & & Gmail & Gmail & Vídeos d'animació \\
\hline & & Twitter & Twitter & $\begin{array}{l}\text { Videojoc Second } \\
\text { Life }\end{array}$ \\
\hline & & Hot potatoes & Wordle & \\
\hline & & Petxa-kutxa & $\begin{array}{l}\text { Wikimedia } \\
\text { Commons }\end{array}$ & \\
\hline & & Cartell en línia & Issuu & \\
\hline & & $\begin{array}{l}\text { Slideshare, fòrum } \\
\text { de debat }\end{array}$ & $\begin{array}{l}\text { Web } \\
\text { d'emmagatzematge }\end{array}$ & \\
\hline & & Wix & Power Point & \\
\hline
\end{tabular}

${ }^{2}$ Nos referimos al concepto de "género" como construcción social que posee cierta estabilidad, pero a la vez es dinámico y cambiante (Camps y Castelló, 2013). En el caso de los géneros académicos que nos ocupan, incluidos en los proyectos de lengua y literatura de secundaria, se refieren a todos aquellos que forman parte de las actividades académicas que se desarrollan en este contexto relacionados con las prácticas de lectura y escritura que predominan en las aulas: géneros literarios, periodísticos, comentarios, resúmenes, reseñas, informes, etc. 


\begin{tabular}{|l|l|l|l|}
\hline & Tuenti & Videoconferència & \\
\hline & & Kahoo it & \\
\hline
\end{tabular}

Observamos un aumento evidente en los últimos cursos de la diversidad de herramientas empleadas por los alumnos, sobre todo plataformas digitales para publicar los trabajos como blogs, wiki, webquest, Slideshare, Issuu; editores de sonido, de vídeos y de fotografía; podcast; videojuegos; videoquiz; videoconferencias; líneas del tiempo; Goggle Maps; Goggle Drive; realización de entradas para Viquipèdia; utilización de las redes sociales $^{3}$.

Se pueden observar en los siguientes ejemplos algunas imágenes donde aparecen TIC empleadas por los alumnos para trabajar diferentes proyectos de lengua y literatura en Secundaria, como una línea del tiempo para trabajar la Renaixença literaria o un mapa de Google para llevar a cabo una ruta literaria.

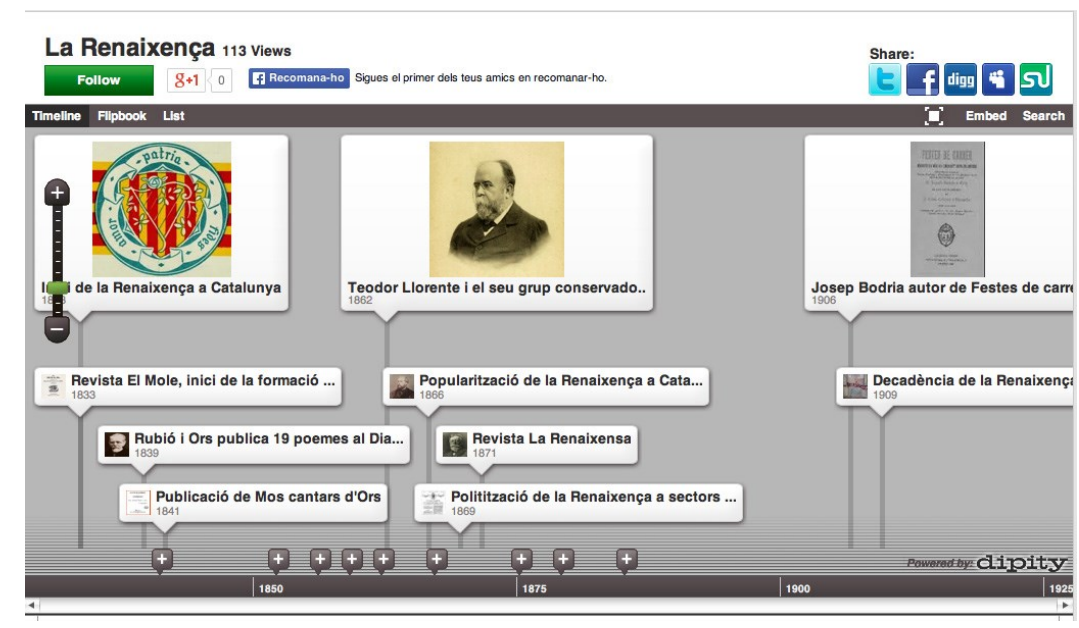

Fig. 1: Linea del tiempo sobre el periodo literario de La Renaixença

\footnotetext{
${ }^{3}$ Además de este recursos, que son los más empleados por el alumnado participante en este estudio, hemos recogido en una web otras recursos que consideramos útiles para mejorar la planificación y el aprendizaje lingüístico ya que en nuestra experiencia como profesoras de secundaria i de universidad los hemos utilizado habitualmente en los proyectos realizados con el alumnado: Del núvol a l'aula.
} 


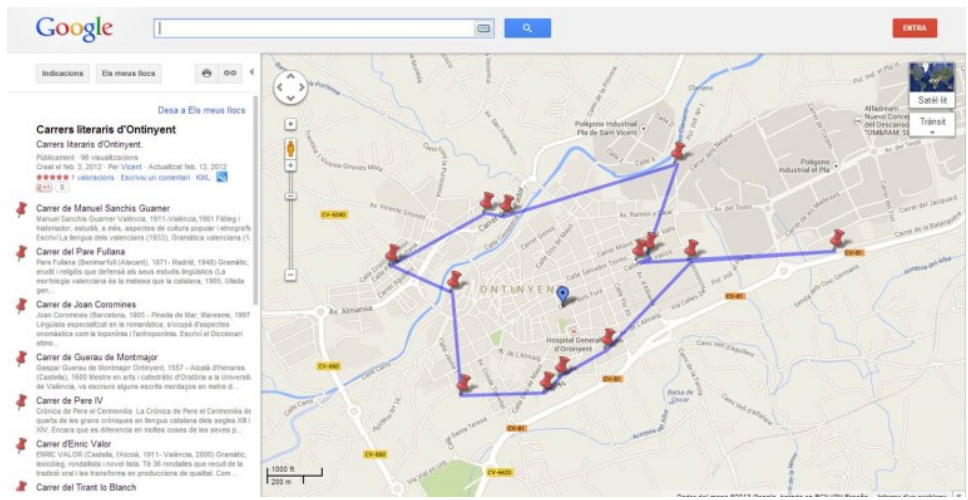

Fig. 2: Ejemplo de la utilización del Google Maps de un grupo de alumnos del máster para indicar una ruta literaria denominada Carrers literaris d'Ontinyent.

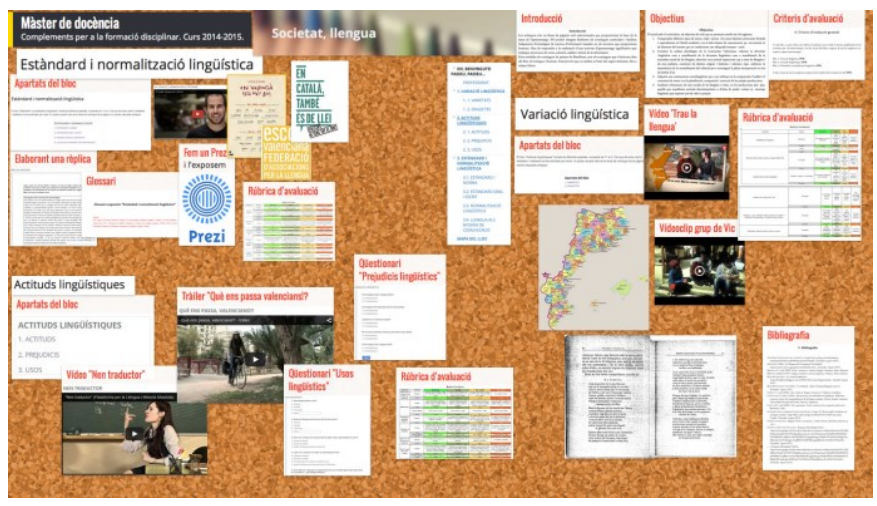

Fig. 3: Ejemplo de presentación de un proyecto de sociolingüistica realizado con un póster en línea con Padlet

\subsection{Ventajas e inconvenientes de las TIC en el trabajo académico}

Según la perspectiva del alumnado del Máster de profesor/a de Secundaria respecto al uso de las TIC en los trabajos académicos, hay más ventajas que inconvenientes y observamos un aumento de las valoraciones positivas en los últimos cursos. Ahora bien, continúa poniéndose en evidencia un déficit en la formación en TIC a pesar del aumento del conocimiento y del uso de las TIC por parte del alumnado. Este déficit en la formación parece que está compensado por el conocimiento y el autoaprendizaje en TIC que realiza una gran parte del alumnado que tiene al alcance ordenadores y dispositivos móviles con aplicaciones que cada vez resultan más accesibles y fáciles de emplear. El déficit de formación no sería el resultado tanto del desconocimiento del funcionamiento de las TIC como de la falta de experiencia para emplearlas con una finalidad didáctica. En este sentido, conviene recordar que hay que partir de lo que es importante que aprenda el alumnado y considerar en qué medida la tecnología ayudará a lograr este propósito, como ya apunta Area (2007) en su decálogo para planificar buenas prácticas docentes con tecnologías. 
Presentamos una muestra de trabajos desarrollados por el alumnado para implementar las TIC en los proyectos de lengua y literatura:

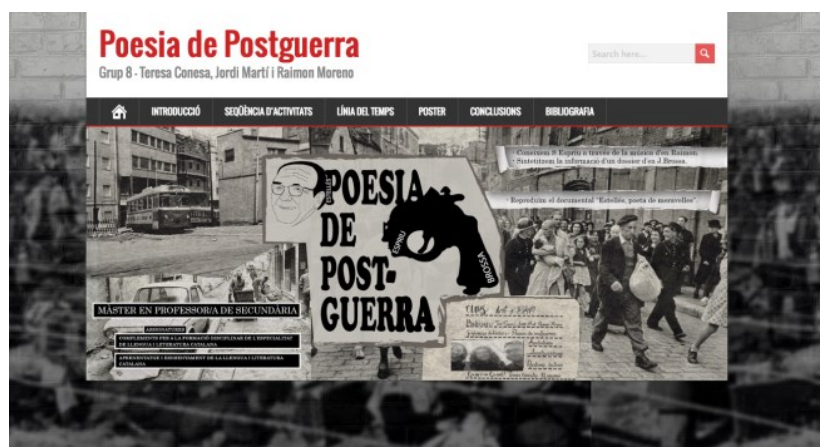

Fig. 4: Ejemplo de una web realizada por los alumnos del máster para trabajar la poesía de posguerra

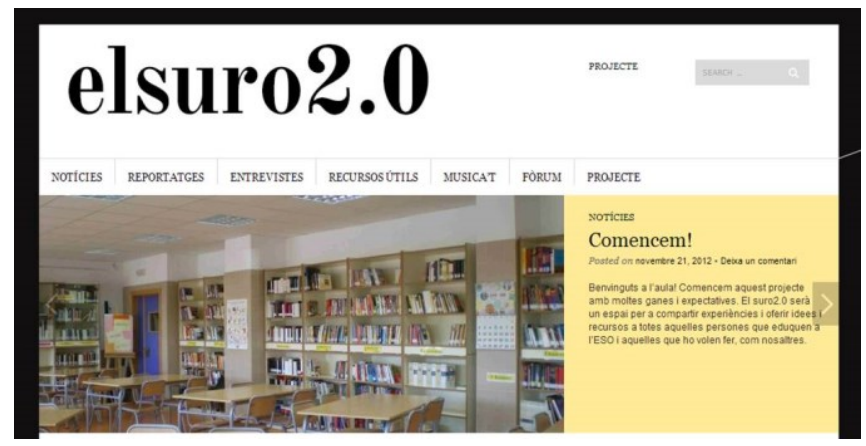

Fig. 5: Periódico digital realizado por los alumnos del máster mediante la creación de una web para trabajar los géneros periodísticos

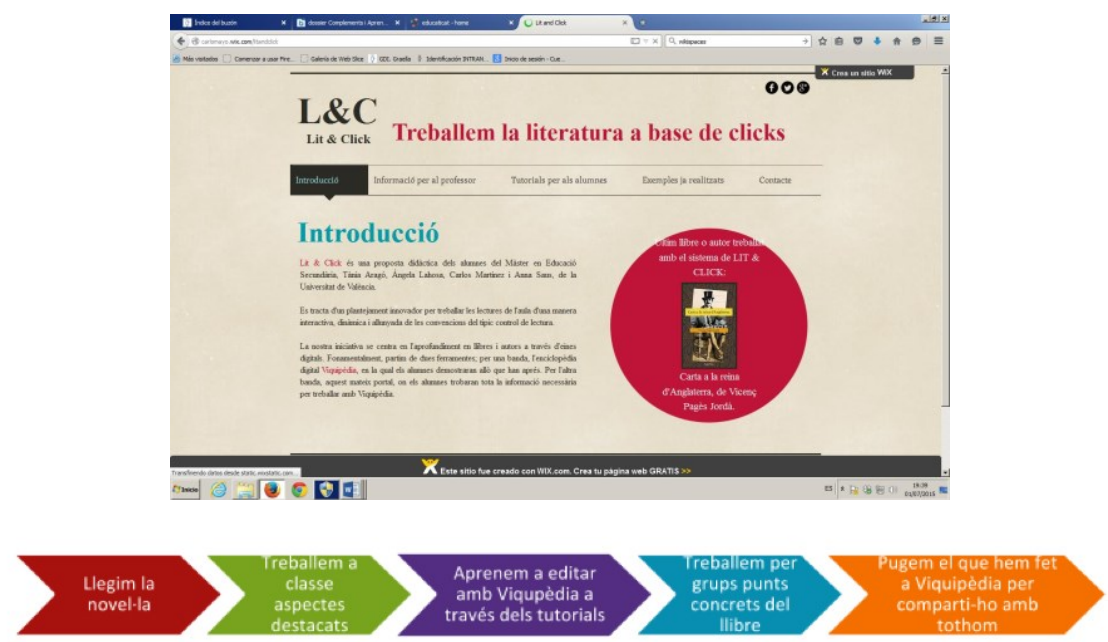

Fig. 6 y 7: Proyecto para trabajar una lectura con la realización de una entrada a Viquipèdia y de una web donde se presentan los tutoriales que tienen que seguir los alumnos

(c)) EY-NC-ND 2017, Universitat Politècnica de València 
En el cuestionario final sobre las ventajas y los inconvenientes de utilizar las TIC en la materia del máster, predominan las ventajas sobre los inconvenientes en todos los cursos y de forma más evidente en los últimos. Las ventajas principales son:

- innovación educativa

- utilidad

- diversidad de maneras de trabajar

- $\quad$ aprender a trabajar con TIC y usar nuevas plataformas

- motivación

- mejora del trabajo en general, especialmente el del profesorado.

Por otro lado, los inconvenientes señalados son:

- tiempo necesario para trabajar con las TIC

- $\quad$ saturación de recursos y exceso de entornos virtuales

- dependencia de la conexión a Internet

- $\quad$ falta de hábitos de uso.

\section{Conclusiones}

En el análisis que hemos realizado centrado en el área de lengua y literatura, los estudiantes han utilizado las TIC de forma ventajosa para la organización de las ideas, la presentación de trabajos académicos, la consulta de recursos lingüísticos en red, la posibilidad de compartir documentos de diferentes formatos y la utilización de sitios web para trabajar en el aula.

La aportación más evidente de las TIC a la escritura académica sería la incorporación de nuevos géneros dentro del ámbito académico que han surgido con la introducción de las TIC en nuestro contexto sociocomunicativo que implican unas nuevas formas de escritura y una inmediatez en la difusión de los textos, como en el caso del post en el Facebook o en el blog, el tuit en el Twitter, el podcast en la radio. Esto comporta que los productos elaborados por los alumnos son más públicos, lo cual supone que se tiene que tener más cuidado en la presentación y, por lo tanto, requiere más dedicación la revisión y la finalización del proyecto. Tal como dice Castelló (2017), se han distinguido diferentes géneros académicos en función de sus objetivos: profesionales (reportajes, noticias, informes), para demostrar conocimientos (exámenes, ejercicios), para manifestar una postura crítica (ensayos, debates), para buscar información (revisiones, estudios), para expresar las propias vivencias (narraciones, diarios, Facebook). Lo importante es que los estudiantes sean capaces de comunicar de manera adecuada lo que han aprendido y tengan al alcance las herramientas adecuadas para conseguirlo.

(cc) EY-NC-ND 2017, Universitat Politècnica de València 
Otra ventaja importante que aportan las TIC, según señalan los mismos alumnos, es favorecer el trabajo colaborativo, la coordinación, la facilidad para compartir. Cómo dice Castelló (2009), es importante favorecer la posibilidad de una escritura colaborativa y que los estudiantes interactúen y reflexionen con las herramientas apropiadas.

En un anterior estudio sobre la utilización de las TIC en trabajos académicos (Martí y Garcia, 2014), ya comentamos que este tipo de recursos permiten desarrollar competencias básicas diversas: la competencia comunicativa, lingüística y audiovisual; la competencia en el tratamiento de la información y la competencia digital; la competencia de aprender a aprender, y la competencia de autonomía y de iniciativa personal. Además, la valoración de los alumnos siempre ha resultado muy satisfactoria dado que el uso de las TIC les motiva a participar en las actividades propuestas en la materia, les facilita acceder a los contenidos del currículum, interaccionar entre ellos y con las profesoras, y compartir otras actividades de interés cultural, referentes musicales y cinematográficos, vídeos, fotografías, etc.

En este análisis hemos visto algunas herramientas TIC que los alumnos consideran útiles para el trabajo en proyectos de lengua y literatura. Sería conveniente, pues, favorecer el conocimiento y el uso de estas herramientas entre el alumnado y el futuro profesorado para mejorar las condiciones de la escritura académica. Los mismos alumnos manifiestan esta carencia de formación en TIC, al tiempo que destacan su utilidad y, por lo tanto, la conveniencia de completar esta formación.

Las conclusiones del estudio muestran que el alumnado cada vez tiene un grado mayor de conocimiento de las TIC, lo cual pone de manifiesto la importancia de implementar más recursos TIC en la docencia con el fin de mejorar la planificación didáctica, la organización de las ideas, la presentación de trabajos académicos, la consulta de recursos en red, la posibilidad de compartir documentos de diferentes formatos y la utilización de sitios web para trabajar en el aula.

Sin embargo, se constata la escasa formación en TIC con finalidades didácticas que demuestra la mayoría de los estudiantes del máster. Por lo tanto, consideramos que es necesario completar la formación universitaria de los futuros docentes en las TIC que se manifiestan más convenientes para favorecer la elaboración de los diferentes géneros académicos, puesto que tienen que saber utilizarlas con solvencia como herramientas de trabajo habituales que les sirvan para aprender a exponer, argumentar, resumir, buscar información, valorar, debatir, etc. Todo ello implica una formación prolongada ya que continuamente aparecen nuevas TIC y se mejoran las anteriores, lo que supone incorporarlas también en el aprendizaje continuo a lo largo de la vida, tanto personal como profesional.

\section{Referencias}

Area Moreira, M. (2007). Decálogo de buenas prácticas con TIC en el aula. $<$ http:/huilliche.blogspot.com/2007/08/declogo-de-buenas-prcticas-con-tic-en.html $>$ [Consulta: 20/10/2016] 
CAmps, A.; CASTElló, M. (2013): "La escritura académica en la Universidad". Revista de Docencia Universitaria, 11 (1), p. 17-36.

CARlino, P. (2013): “Alfabetización acadèmica diez años después". Revista Mexicana de Investigación Educativa, 57, p. 355-381.

CAStelló, M. (2014): "Los retos actuales de la alfabetitzación acadèmica: estado de la cuestión y últimas investigaciones" en Enunciación, 19 (2), p. 346-365.

CASTElló, M. (2017): “Tot mirant-se al mirall”. Articles de Didàctica de la Llengua i la Literatura, 73, p. 7-13.

Martí Climent, A.; Garcia Vidal, P. (2014). "El treball per projectes amb les TIC a l'àrea de Llengua i Literatura. Una experiència didàctica en la formació del professorat de Secundària" en Articles de Didàctica de la Llengua i la literatura, 64. Disponible en:

$<$ https://www.google.es/search?q=el+treball + per+projectes + amb + les + TIC $+\mathrm{a}+1 \% 27 \% \mathrm{C} 3 \% \mathrm{~A} 0 \mathrm{rea}+\mathrm{de}+$ llengua $+\mathrm{i}+$ literatura $\& \mathrm{ie}=$ utf- 8 \&oe $=$ utf- $8 \&$ client $=$ firefox-

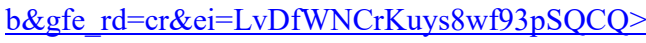

